\title{
Hoher Qualitätsstand in Schweizer Ärztenetzen
}

Wolfgang Czerwenka ${ }^{a}$,

Karl Metzger ${ }^{b}$, Jörg Fritschic ${ }^{c}$

a Vorsitzender Medizinische Geschäftsleitung argomed Ärzte AG, Arbeitsgruppe Quali-med-net

b Leiter Managed Care BlueCare, Arbeitsgruppe Quali-med-net

c Präsident medswiss.net
Korrespondenz: medswiss.net - Schweizer Dachverband der Ärztenetze Grütlistrasse 36

CH-8002 Zürich

Tel. 0442804405

Fax 0442804403

medswiss.net@hin.ch

\begin{abstract}
Einleitung
Von integrierten Versorgungsnetzen [1] wird neben einer besseren Qualität der Versorgung auch ein kostendämpfender Beitrag in den wachsenden Gesundheitskosten durch Übernahme einer ökonomischen Mitverantwortung der Leistungserbringer erwartet [2]. Im Zuge dieser Entwicklung stellt die Erhebung und Darstellung der Versorgungsqualität in Ärztenetzen eine besondere Herausforderung dar. Der Dachverband der Schweizer Ärztenetze medswiss.net stellt seit 2005 mit Quali-med-net - einem spezifischen Qualitätsindikatorenset für Ärztenetze - ein Instrument zur Qualitätsentwicklung und Selbstevaluation zur Verfügung [3]. Ende 2009 hat medswiss.net erstmalig eine Erhebung über die Anwendung der Quali-mednet-Indikatoren bei seinen Mitgliedernetzen durchgeführt. Mit 19 Ärztenetzen haben sich 42\% der Mitgliedernetze von medswiss.net einer Selbstevaluation unterzogen. Mit dieser Datengrundlage sind erstmals Aussagen zum Entwicklungsstand der Qualitätsarbeiten in Schweizer Ärztenetzen möglich: Im Mittelwert
\end{abstract}

\section{Abbildung 1}

Erfüllungsgrad pro Quali-med-net-Indikator über alle 19 Ärztenetze hinweg.

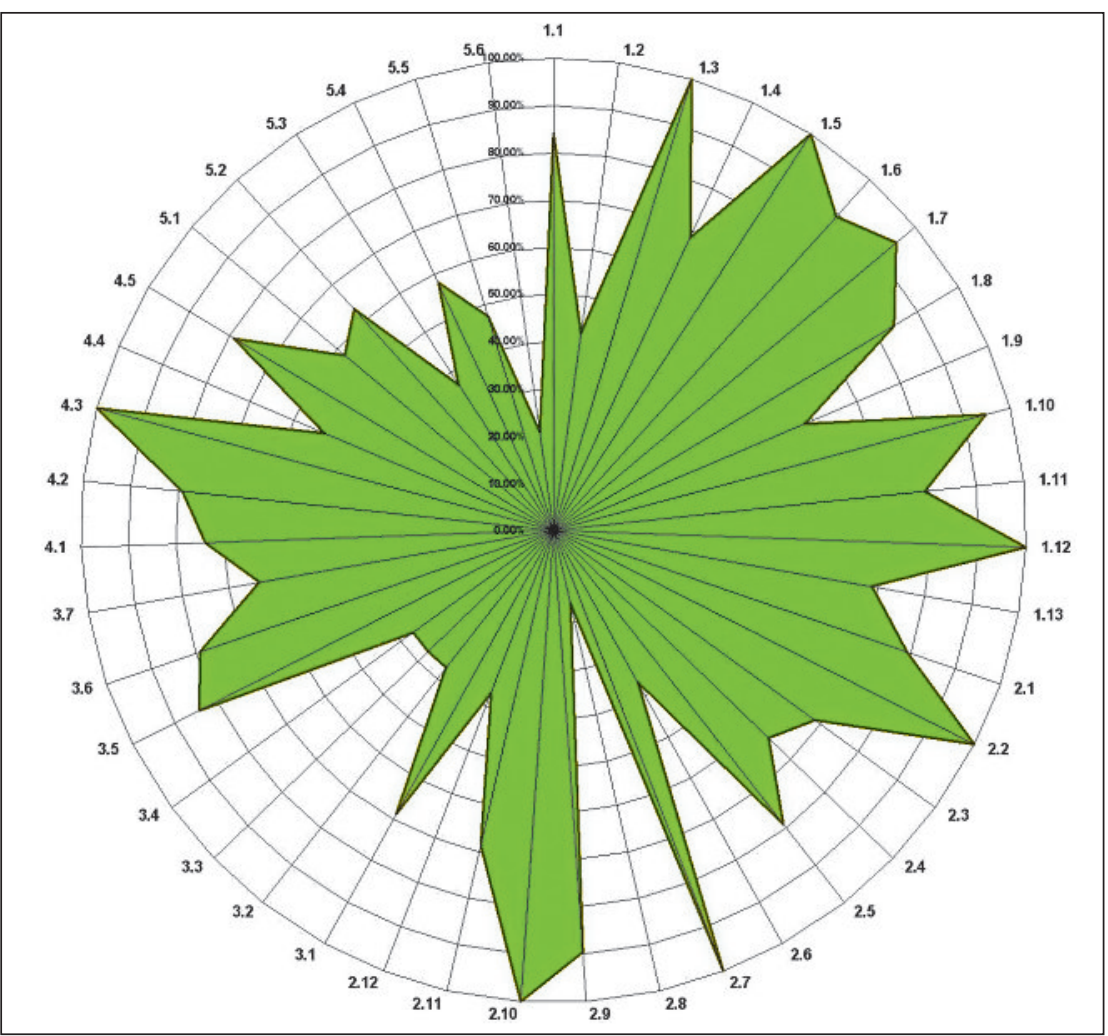

erfüllen rund 70\% der teilgenommenen Ärztenetze die 43 Quali-med-net Indikatoren. Dies bestätigt den hohen Qualitätsstand in den Schweizer Ärztenetzen.

\section{Zielsetzung und Aufbau des Quali-med-net- Indikatorensets}

Das Indikatorenset Quali-med-net wurde 2005 durch die Arbeitsgruppe [4] Qualität von medswiss.net entwickelt. Quali-med-net ist als Selbstevaluationsinstrument aufgebaut und ist in fünf Qualitätsdimensionen mit insgesamt 43 Indikatoren gegliedert (siehe Tabelle 1).

- Mitgliedschaft, Organisation und Infrastruktur: 13 Indikatoren

- Interne und externe Zusammenarbeit: 12 Indikatoren

- Prozess-Management: 7 Indikatoren

- Kommunikation: 5 Indikatoren

- Outcome: 6 Indikatoren

Quali-med-net ist allen Ärztenetzen von medswiss. net zugänglich und soll durch die Darstellung der Vielzahl an Leistungen und Entwicklungsprozessen in einem Ärztenetz primär als Auswahl- und Orientierungshilfe dienen. Damit bietet Quali-med-net mit seinem Bottom-up-Ansatz die Basis für qualitätsfördernde Massnahmen und Entwicklungen als lernendes System.

Je Indikator ist eine Messgrösse, ein Standard und ein Weiterentwicklungsvorschlag definiert (siehe Beispiel Tabelle 2). 2007 wurde Quali-med-net für Ärztenetze weiterentwickelt, indem ausgehend von den 43 Indikatoren 15 Top-Qualitätsindikatoren ausgewählt wurden für die zukünftige mögliche Vergabe eines Labels.

\section{Erste umfassende Erhebung}

Im Zeitraum von Juli bis Oktober 2009 wurde erstmals eine umfassende Erhebung bei den Ärztenetzen von medswiss.net durchgeführt. medswiss.net hat dazu seinen damals 45 Mitgliedernetzen den Selbstevaluationsbogen zugestellt. 19 Ärztenetze haben sich an der Erhebung beteiligt, was einer guten Rücklaufquote von $42 \%$ entspricht. Damit liegen erstmals detaillierte Daten zum Entwicklungsstand der Schweizer Ärztenetze vor. Die Ergebnisse der Erhebung wurden durch medswiss.net aufbereitet und den teilnehmenden Ärztenetzen zur Verfügung gestellt. Zusätzlich erhielt jedes Ärztenetz ein anonymisiertes Benchmarking über alle an der Erhebung beteiligten Ärztenetze. Damit konnte jedes Ärztenetz seine eige- 


\section{Tabelle 1}

Die 43 Quali-med-net-Indikatoren.

\section{Mitgliedschaft, Organisation und Infrastruktur}

1.1 Aus-, Fort- und Weiterbildung Netzarzt*

1.2 Aus-, Fort- und Weiterbildung nichtärztliches Praxispersonal

1.3 Beitritts- und Mitgliedschaftskriterien

1.4 Leitbild* $^{*}$

1.5 Netzwerkführung*

1.6 Mitgliederwesen

1.7 Elektronische Vernetzung*

1.8 Eigenes Daten-Informationssytem

1.9 Serviceleistungen für Netzärzte

1.10 Netzwerk Rechnungswesen

1.11 Sponsoring und Zuwendungen

1.12 Notfallmanagement

1.13 Anlauf- und Ombudsstelle Patienten*

2. Interne und externe Zusammenarbeit

2.1 Zusammenarbeitsrichtlinien netzintern*

2.2 Qualitätszirkel-Arbeit*

2.3 Einführung/Begleitung neuer Netzärzte

2.4 Netzwerkspezifische Ausbildungsangebote für nichtärztliches Praxispersonal

2.5 Zusammenarbeit mit netzexternen Spezial- und Spitalärzten*

2.6 Zusammenarbeit weitere Leistungserbringer

2.7 Zusammenarbeit andere Ärztenetze

2.8 Zusammenarbeit Patientenorganisationen

2.9 Zusammenarbeit Gesundheitspolitik/Presse

2.10 Zusammenarbeit Krankenversicherer

2.11 Zusammenarbeit Ausbildungsinstitutionen

2.12 Zusammenarbeit Forschungszentren

3. Prozess-Management

3.1 Medizinisches Überweisungsmanagement

3.2 Medikamentenoptimierung

3.3 Praxisevaluation*

3.4 Medizinische Präventionsmassnahmen

3.5 Datenauswertungen*

3.6 Patientensicherheit/CIRS*

3.7 Guidelines*

4. Kommunikation

4.1 Informationen für Patienten, Netzärzte und Partner

4.2 Website

4.3 Netzwerkveranstaltungen

4.4 Netzinternes Konflikt- und Krisenmanagement

4.5 Datenschutz und Datensicherheit

5. Outcome

5.1 Klinische Projekte und Studien

5.2 Publikationen

5.3 Patientenbefragungen

5.4 Umfragen und Erhebungen bei Netzärzten

5.5 Case und Disease Management

5.6 Effizienz- und Wirtschaftlichkeitsstudien

* 15 Quali-med-net-Topindikatoren

nen Qualitätsanstrengungen einordnen und erhielt einen Quervergleich.

\section{Hoher Erfüllungsgrad in den Ärztenetzen}

Die Erhebung sollte den Ärztenetzen neben der eigenen Standortbestimmung einen Vergleich der eigenen Qualitätsentwicklung gegenüber dem Entwicklungsstand anderer Ärztenetze erlauben. Dazu musste durch das Ärztenetz für jeden Quali-med-net-Indikator angegeben werden, ob dieser gemäss Definition er- füllt ist oder nicht. Damit konnte bei der Auswertung der Selbstdeklaration je Ärztenetz der Erfüllungsgrad je Indikator bestimmt werden.

Über alle 43 Indikatoren hinweg betrachtet wurde von den 19 Ärztenetzen ein Erfüllungsgrad im Mittel von insgesamt 69,2\% erreicht (Median $=67,4 \%$ ). Insgesamt wurden 7 Indikatoren wie zum Beispiel das Vorhandensein eines Qualitätszirkels von allen 19 Ärztenetzen zu 100\% erfüllt (siehe Abb. 1). Diese hohen Werte bestätigen auch die neuesten Zahlen der Ärztenetz-Erhebung 2010 des Forums Managed Care [5].

Bezogen auf die fünf Qualitätsdimensionen variieren die Werte im Mittel von 47,4\% bis 81,8\%.

- Mitgliedschaft, Organisation und Infrastruktur: $81,8 \%$ (Median $=84,6 \%$ )

- Interne und externe Zusammenarbeit:

$69,7 \%$ (Median $=75 \%)$

- Prozess-Management: 57,9\% (Median = 57,1\%)

- Kommunikation: 76,8\% (Median $=80 \%$ )

- Outcome: 47,4\% (Median = 33,3\%)

Die insgesamt erfreulich hohen Werte zeigen das zusätzliche Engagement der Ärztenetze in der Qualitätsarbeit. Da es sich um eine freiwillige Teilnahme mit Selbstdeklaration handelte, kann ein Selektionsbias nicht ausgeschlossen werden.

\section{Grosse Varianz unter den Ärztenetzen}

Die Erhebung zeigt aber auch, dass der Erfüllungsgrad je Ärztenetz stark variieren kann: Der tiefste Erfüllungsgrad eines Ärztenetzes liegt im Mittel über alle Indikatoren bei 46,5\%, der höchste bei 97,7\% (siehe Abb. 2). Betrachtet man die einzelnen fünf Qualitätsdimensionen, liegt die Varianz im Mittel sogar zwischen $15 \%$ und $100 \%$ je nach Ärztenetz. Dies spiegelt deutlich den unterschiedlichen Reifegrad oder die Schwerpunktsetzungen der Qualitätsarbeit in den einzelnen Ärztenetzen.

\section{Wertung der Ergebnisse}

Qualitätsarbeit ist eine ständige Aufgabe für Ärztenetze. Noch keines der teilnehmenden Ärztenetze erfüllt bislang alle 43 Quali-med-net-Indikatoren vollumfänglich. Die drei Ärztenetze mit den höchsten Werten kommen aber auf einen beachtlichen Erfüllungsgrad von 86 bis $98 \%$.

Konzentriert man sich bei der Auswertung auf die 15 Top-Indikatoren, beträgt der Erfüllungsgrad über alle Ärztenetze bereits im Mittel 76,5\% (Median $=80 \%$ ), wobei drei Ärztenetze sogar $100 \%$ erreichen.

Die Erhebung hat gezeigt, dass Quali-med-net als Selbstevaluationsinstrument einem klaren Bedürfnis entspricht. Ärztenetze können damit ihre Qualitätsarbeit transparent und klar strukturiert erheben. Diese Transparenz können die Ärztenetze zum einen als Standortbestimmung und zur Weiterentwicklung der eigenen Qualitätsarbeit nutzen, zum anderen aber auch gegen aussen ihr Qualitätsprofil gegenüber ihren Partner darstellen und kommunizieren. 
Tabelle 2

Beispiel Quali-med-net-Indikator 2.1

\begin{tabular}{llll} 
Indikator & Messgrösse & Standard & Entwicklung/Ausbau \\
\hline Zusammen- & Richtlinien & Regelung von & Kontinuierliches \\
arbeitsrichtlinien & vorhanden & Vertretungen, & Schnittstellen- \\
netzintern & Ja/Nein & $\begin{array}{l}\text { Notfalldienst, Über- } \\
\text { und Rücküberweisungen }\end{array}$ & management \\
mit Netzärzten & & $\begin{array}{ll}\text { innerhalb } \\
\text { des Ärztenetzes }\end{array}$ & \\
& & &
\end{tabular}

Abbildung 2

Erfüllungsgrad über alle 43 Quali-med-net-Indikatoren pro Ärztenetz.

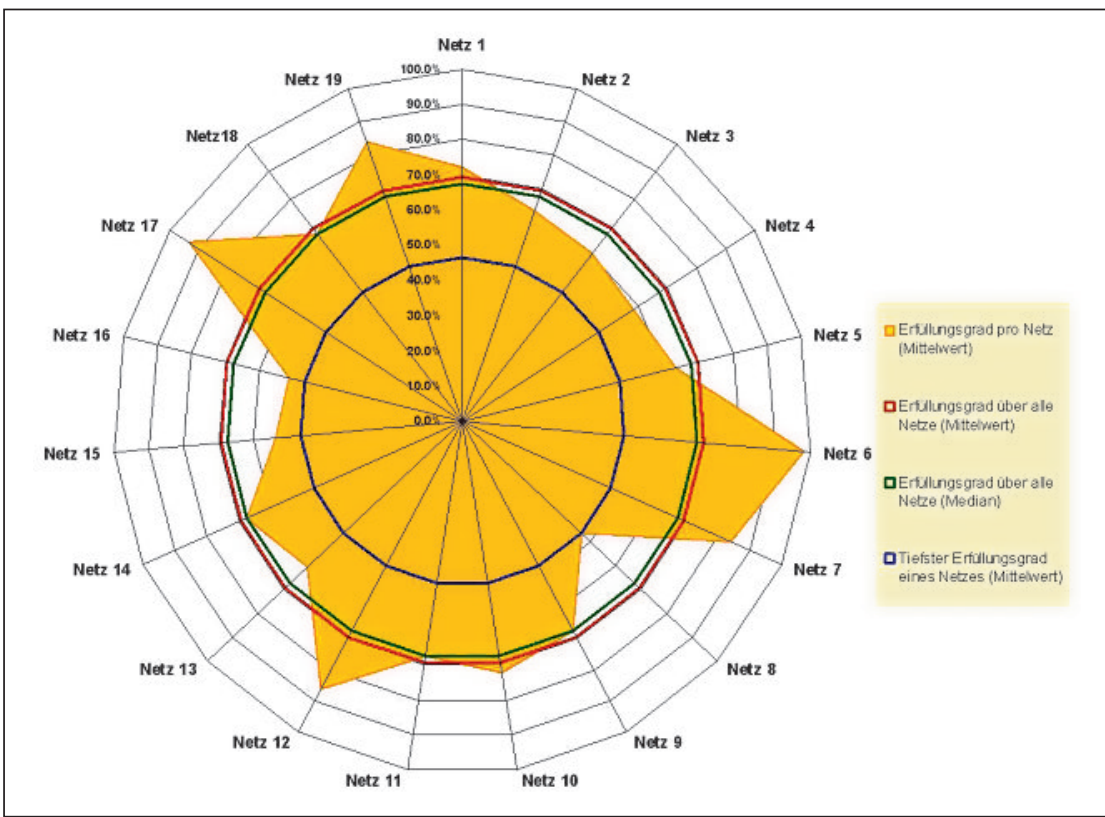

\section{Ausblick}

Mit Quali-med-net wurde ein weiterer wichtiger Schritt in der Qualitätsförderung und -erhebung in den Ärztenetzen gemacht. Quali-med-net wird sich weiterentwickeln und der Vorstand von medswiss.net sieht folgende Schwerpunkte:

- medwiss.net unterstützt die Anwendung und Verbreitung des Indikatorensets in den Ärztenetzen, welche die Einführung von Quali-med-net planen, durch spezifische Beratungsangebote.

- Einzelne Indikatoren können für eine eindeutigere Definition in Zukunft noch ergänzt oder geschärft werden.
- Die auf Selbstdeklaration basierte Erhebung soll periodisch (z. B. alle 2 bis 3 Jahre) von medswiss.net durchgeführt werden, damit der eigene Fortschritt in den Ärztenetzen ersichtlich wird und in Zukunft auch longitudinale Vergleiche möglich werden.

- Daneben soll das Indikatorenset von Quali-mednet gezielt ausgebaut und bei den Ärztenetzen etabliert werden. Für die Verantwortlichen ist in Zukunft auch die Einführung eines Quali-mednet Labels mit externer Zertifizierung denkbar.

\section{Literatur}

1 Forum Managed Care. Orientierungspapier Integrierte Versorgung in der Schweiz. Care Management. 2009;2(3):1-4.

2 Fritschi J. Budgetmitverantwortung und KVG-Revision Managed Care / Integrierte Versorgung. Schweiz Ärztezeitung. 2010;91(33):1218-9.

3 Czerwenka W, Metzger K. Förderung und Bewertung der Qualität in Ärztenetzwerken mit dem Indikatorenset Quali-med-net. Schweiz Ärztezeitung. 2006;87(51/52): 2213-5.

4 Arbeitsgruppe Quali-med-net: Alex Buchli (SGAM), Peter Christen (zmed/VHZ), Wolfgang Czerwenka (argomed), Rainer Hurni (zmed/VHZ), Christof Kaech (Ärztenetz Biel-Seeland), Karl Metzger (BlueCare), Andreas Schneider (eastcare), Hans Beat Stiefel (hawadoc)

5 Berchtold P et al. Ärztenetze in der Schweiz 2010: Starkes Wachstum. Care Management. 2010;3(3): 45-6.

Verband der Schweizer Ärztenetze medswiss.net Der Verband umfasst aktuell 47 Hausarztvereine und Ärztenetze mit rund 2000 Ärztinnen und Ärzten, die ca. 600000 Managed-Care-Versicherte betreuen.

Im Namen des Schweizer Dachverbandes der Ärztenetze dankt der Vorstand allen Mitgliedern der Arbeitsgruppe Quali-med-net für die Erarbeitung dieses wegweisenden und umfassenden Qualitätsinstrumentes. Quali-med-net ist eine Dienstleistung des Dachverbandes für seine Mitgliedernetze. Das Instrument ermöglicht den angeschlossenen Ärztenetzen, mit vertretbarem Aufwand den eigenen Qualitätsstand darzustellen. Quali-med-net ist damit auch eine Basis der Qualitätsentwicklung für eine integrierte Versorgung. 